\title{
Sharp fluorescence nanofiber network of CdSe/CdS core-shell nanoplatelets in polyvinylpyrrolidone
}

\author{
Dongni Han ${ }^{\mathrm{a}}$, Lanlan Yang ${ }^{\mathrm{b}}$, Zhongyi $\mathrm{Hu}^{\mathrm{a}}$, Zhonglin Du ${ }^{\mathrm{a}}$, Yao Wang ${ }^{\mathrm{a}}$, Ze Yuan ${ }^{\mathrm{a}}$, Qiao Wang ${ }^{\mathrm{a}}$, \\ Mikhail Artemyev ${ }^{\mathrm{b}}$, Jianguo Tang, ${ }^{\mathrm{a}, *}$ \\ ${ }^{a}$ Institute of Hybrid Materials, National Center of International Joint Research for Hybrid Materials \\ Technology, National Base of International Science \& Technology Cooperation on Hybrid Materials, \\ Qingdao University, Qingdao 266071 China \\ b Research Institute for Physical Chemical Problems of the Belarusian State University, Minsk 220006 \\ Belarus
}

*Corresponding author, e-mail: tang@qdu.edu.cn

Received 5 Jun 2020

Accepted 31 Aug 2020

\begin{abstract}
In this work, a novel nanofiber network of colloidal semiconductor CdSe/CdS nanoplatelets (NPLs)/polyvinylpyrrolidone (PVP) with characteristics of porous membranes has successfully been fabricated via the electrospinning technique, in which NPLs and PVP were used as fluorescence agent and fiber matrix, respectively. Firstly, the CdSe core NPLs with quasi 2D geometry was synthesized, and the epitaxial growth of the CdS shells was performed by the atomic layer deposition (c-ALD) method at room temperature. CdSe/CdS core-shell NPLs with a narrow, ca. $20 \mathrm{~nm}$ photoluminescence band were blended with PVP dissolved in ethanol-trichloroethylene mixture; and hybrid polymer-NPLs fibers were obtained through the electrospinning technique. The final nanofiber network exhibits the excellent optical properties of the NPLs, which has potential for separation and absorption of pollutants and isolation of microorganisms.
\end{abstract}

KEYWORDS: semiconductor nanoplatelets, polymer, electrospinning, hybrid materials, fluorescence nanofiber network

\section{INTRODUCTION}

In recent years, microporous membranes have attracted the attentions for separation and absorption of pollutants and for isolation of microorganisms [1]. The latest publications [2] indicated the possibility that this structure can be fabricated from nanofiber networks, with the advantages of controllable porous size from tens of nanometers to micrometers [3]. In the past decade, there are publications on the methodology, whereas [4], it is not possible to know the degree of pollution, harmfulness or toxicity through this kind of porous structure. Thus, it is necessary to design a structure that can impart this microporous structure to sense these dangerous matters. Thus, fluorescence sensing is ideal strategy to meet this task [5].

Colloidal semiconductor quantum dots (QDs) are the promising next-generation units for the application advantages of cost-effective production, superior color purity, high quantum yield (QY), and precisely tunable emission wavelength through the whole visible to the near infrared range [6-9]. Such advantages have encouraged much efforts to de- velop sensing applications [10]. However, spherical CdSe QDs suffer from the size sensitivity to broaden spectra and to shift emission wavelengths with the change of their diameter scale and distribution [11]. Whereas, CdSe nanoplatelets (NPLs) have zero size deviation along their normal direction along which the quantum confinement occurs [12]. Therefore, CdSe NPLs demonstrate narrower absorption and emission bands. As compared to QDs, CdSe NPLs can potentially exhibit weaker Coulomb interaction between carriers, which allows for more efficient charge injection without affecting confinement and recombination regimes $[13,14]$.

Electrospinning is an effective and low-cost technology for preparing polymer fibers with diameter range from tens of nanometers to a few micrometers $[15,16]$. The high surface-to-volume ratio of electrospun fibers has encouraged extensively on tissue engineering scaffolds, energy storage, sensors and drug delivery [17-20]. The sensitive 2,4-dinitrotoluene fluorescence sensors based on porous electrospun fibres and porous membranes prepared from pyrene-doped poly (methyl methacrylate), polyvinyl chloride, polystyrene, and 
co-polymers from these polymers have been successfully developed [21]

So far, there are just few studies on QD$\mathrm{s} /$ polymer composite fibers by direct mixing method $[22,23]$. And recently, the ultrafine fibers of the QDs doped in polymer hosts illustrated successes of electrospinning method for electronic and optoelectronic devices [24,25]. Reports indicated that there are two methods to introduce QDs into polymer fibers, i.e. in situ formation method and direct blending [26]. Although the in situ formation method has a precise control of the size distribution, the formation process usually leads to increases of surface defects resulting in poor optical properties [27]. The QDs/polymer direct mixing method can solve the problems mentioned above and achieve ultrafine fibers with excellent optical properties and narrow size distribution [28, 29].

Herein, novel hybrid luminescent nanofiber network based on the CdSe/CdS coreshell NPLs/polyvinylpyrrolidone (PVP) was demonstrated. The NPLs have been introduced into the PVP solution by addition of trichloroethylene to the polymer solution. Compared with similar work in the past, this new type of network was doped with NPLs that have high quantum yield (QY) and extremely narrow full-width at half-maximm (FWHM). The different novel hybrid luminescent nanofiber network maintains sharp fluorescence as usual after electrospinning. This work provided a new hybrid nanofiber network with great potential applications for sensing and detecting.

\section{MATERIALS AND METHODS}

\section{Materials}

Cadmium acetate dehydrate (Cd(OAc) $)_{2} \cdot 2 \mathrm{H}_{2} \mathrm{O}$, 99.99\%), ammonium sulfide $\left(\left(\mathrm{NH}_{4}\right)_{2} \mathrm{~S}, 40 \%\right)$, myristic acid, selenium powder (Se, 99.99\%), 1-octadecene (1-ODE, 90\%), oleylamine (OAm, 80-90\%), oleic acid (OA, 90\%), and PVP (Mw $=1300000 \mathrm{~g} / \mathrm{mol}$ ) were purchased from Aladdin, Shanghai, China. Methanol, hexane, isopropanol, ethanol, trichloroethylene $\left(\mathrm{C}_{2} \mathrm{HCl}_{3}\right)$, toluene, and acetonitrile were from Sinopharm Chemical Reagent Co., Ltd, Shanghai, China. Nmethylformamide (NMF, 99\%) was from Macklin, Shanghai, China. All chemicals were used as received and without further purification.

\section{Characterization}

UV-vis absorption of NPLs solution was recorded by PerkinElmer Lambda 750S spectrophotometer in the range of $200-2300 \mathrm{~nm}$ using a glass cuvette with a $1 \mathrm{~mm} \times 1 \mathrm{~mm}$ optical path. Photoluminescence (PL) spectra and fluorescent optical microscopic photographs were measured with Cary Eclipse fluorescent spectrometer and OLYMPUS BX41 universal microscope with a UV lamp emitting in the wavelength range of 340-380 nm, respectively. The morphologies of NPLs and hybrid fibers were characterized by transmission electron microscopy (TEM, JEM$2000 \mathrm{Ex}$ ) and scanning electron microscopy (SEM, Tescan-Vage3). Fourier transform infrared (FTIR) spectra were recorded on a Nicolet 5700 infrared spectrophotometer in the range of $4000-400 \mathrm{~cm}^{-1}$.

\section{Preparation of CdSe and CdSe/CdS NPLs}

Twenty mmol of $\mathrm{Cd}(\mathrm{OAc})_{2} \cdot 2\left(\mathrm{H}_{2} \mathrm{O}\right)$ was dissolved in $50 \mathrm{ml}$ of methanol and mixed with $45 \mathrm{mmol}$ of myristic acid dissolved separately in $300 \mathrm{ml}$ of methanol. The white precipitate was filtered out from the solution and washed with methanol three times to remove the excess precursors. The final $\mathrm{Cd}(\mathrm{myr})_{2}$ powder was dried under vacuum at $40^{\circ} \mathrm{C}$ overnight. Then, $\mathrm{Cd}(\mathrm{myr})_{2}$ (140 mg), Se powder $(24 \mathrm{mg})$ and 1-ODE $(15 \mathrm{ml})$ were introduced into a three-neck round-bottom flask and degassed under vacuum at $100^{\circ} \mathrm{C}$ for $30 \mathrm{~min}$ with stirring. After degassing, the reaction mixture was heated up to $240^{\circ} \mathrm{C}$ under nitrogen atmosphere. When the temperature reached $195^{\circ} \mathrm{C}, 80 \mathrm{mg}$ of $\mathrm{Cd}(\mathrm{OAc})_{2} \cdot 2\left(\mathrm{H}_{2} \mathrm{O}\right)$ were rapidly added into the reaction mixture. After the temperature reached $240^{\circ} \mathrm{C}$, the reaction mixture was stirred for $8 \mathrm{~min}$, then $2 \mathrm{ml}$ of OA were added and the solution cooled down to $80^{\circ} \mathrm{C}$. To overgrow CdSe core NPLs with CdS shell by ADL method [30], $1 \mathrm{ml}$ of CdSe cores in $4 \mathrm{ml}$ hexane dissolved in $3 \mathrm{ml}$ hexane, and the first monolayer was deposited by adding $12.5 \mu \mathrm{l}$ of $\left(\mathrm{NH}_{4}\right)_{2} \mathrm{~S}$ in $5 \mathrm{ml} \mathrm{NMF}$ and vigorously stirring until complete the phase transfer of NPLs from hexane to NMF phase. The S-coated CdSe NPLs were precipitated from NMF by adding a mixture of toluene and acetonitrile, centrifuged at $10000 \mathrm{rpm}$ and redispersed in $5 \mathrm{ml}$ of NMF. Then, the monolayer of $\mathrm{Cd}$ was deposited by introducing $1.5 \mathrm{ml}$ of $0.25 \mathrm{M}$ solution of $\mathrm{Cd}(\mathrm{OAc})_{2} \cdot 2\left(\mathrm{H}_{2} \mathrm{O}\right)$ in NMF, the mixture was stirred for $3 \mathrm{~min}$, after which the CdSe/CdS coreshell NPLs were precipitated by adding the mixture of toluene and acetonitrile (toluene:acetonitrile $=1: 1, \mathrm{v} / \mathrm{v}$ ). To further increase the CdS shell thickness, the above procedure was repeated three times and the final core-shell NPLs with different thickness were dispersed in trichloroethylene with the addition of OAm. 


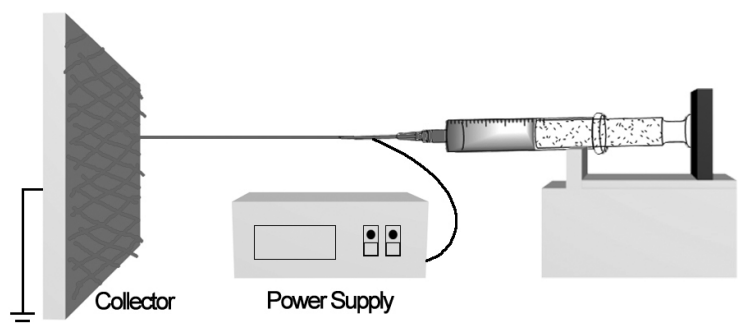

Fig. 1 Schematic diagram of the electrospinning process.

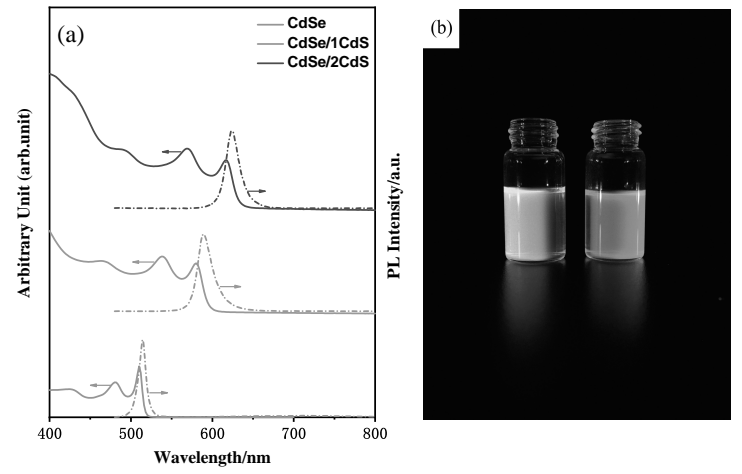

Fig. 2 (a) Absorption and photoluminescence spectra of CdSe core and CdSe/CdS core-shell NPLs with $1 \mathrm{ml}$ and $2 \mathrm{ml}$ thick CdS shell in hexane; (b) sample picture of CdSe core and CdSe/CdS core-shell NPLs. The photoluminescence spectra are obtained by excitation at $460 \mathrm{~nm}$.

\section{Fabrication of NPLs/PVP hybrid nanofiber network}

To prepare the polymer solution, $10 \mathrm{~g}$ PVP was dissolved in the mixture of ethanol and $\mathrm{C}_{2} \mathrm{HCl}_{3}$ (ethanol: $\mathrm{C}_{2} \mathrm{HCl}_{3}=2: 1, \mathrm{v} / \mathrm{v}$ ) and stirred for $12 \mathrm{~h}$ to obtain a homogeneous solution of $10 \mathrm{wt} \% \mathrm{PVP}$ solution. Then, different contents of NPLs were added into the PVP solution and the mixture was continuously and vigorously stirred and sonicated strongly to avoid aggregation of NPLs. The asprepared solutions with different contents ( $1 \mathrm{wt} \%$, $3 \mathrm{wt} \%$ and $5 \mathrm{wt} \%$ ) of NPLs were loaded into $5 \mathrm{ml}$ plastic syringes attached with stainless steel needles. During the electrospinning process, using aluminum foil as the positive electrode collector, the applied voltage was $10 \mathrm{kV}$ and the distance between the spinneret tip and the collector was kept at $20 \mathrm{~cm}$. All of these processes were demonstrated under ambient conditions (Fig. 1).

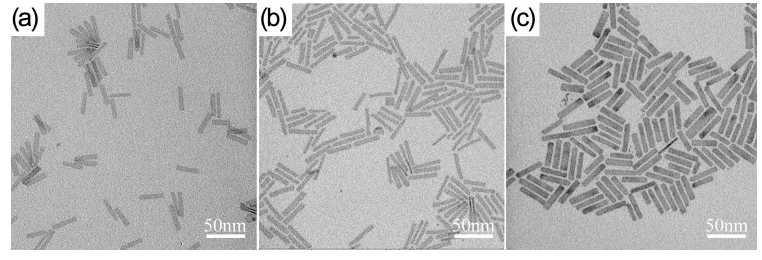

Fig. 3 TEM images of CdSe core and CdSe/CdS coreshell NPLs: (a) core CdSe NPLs, (b) CdSe/1CdS NPLs, (c) CdSe/2CdS NPLs.
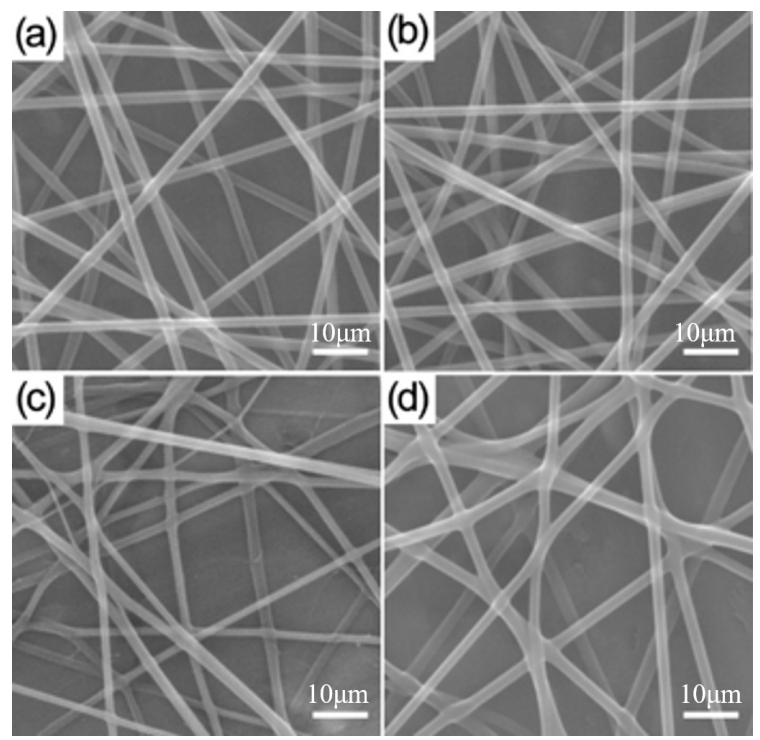

Fig. 4 SEM images of nanofiber network with different NPLs contents: (a) $0 \mathrm{wt} \%$, (b) $1 \mathrm{wt} \%$, (c) $3 \mathrm{wt} \%$, (d) $5 \mathrm{wt} \%$.

\section{RESULTS AND DISCUSSION}

Fig. 2(a) shows the absorption and emission spectra of as-prepared CdSe core, CdSe/1CdS and Cd$\mathrm{Se} / 2 \mathrm{CdS}$ core-shell NPLs in hexane solutions at the room temperature. During deposition of CdS shell, the heavy-hole absorption peak shifts from $\lambda=$ $510 \mathrm{~nm}$ to $617 \mathrm{~nm}$ after $2 \mathrm{c}$-ALD cycles, while the stokes shift increases from $4 \mathrm{~nm}$ to $7 \mathrm{~nm}$. Introduction of CdS shell resulted in broadening of PL spectrum to a FWHM $\approx 20 \mathrm{~nm}$. The PL QYs of the CdSe/1CdS and CdSe/2CdS core-shell NPLs were about $20 \%$ and $40 \%$, respectively. Fig. 2(b) is the sample picture, the left one is the CdSe core and the right one the CdSe/CdS core-shell NPLs.

The geometric morphology of the NPLs before and after shell growth was characterized by TEM. Fig. 3 shows the TEM images of core and core-shell NPLs. TEM images show that CdS shell uniformly 


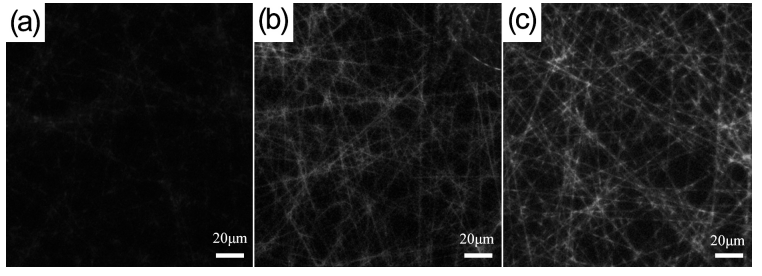

Fig. 5 Microscopy images (under the UV light) of CdSe/1CdS NPLs/PVP nanofiber network with different NPLs contents: (a) $1 \mathrm{wt} \%$, (b) $3 \mathrm{wt} \%$, (c) $5 \mathrm{wt} \%$. White scale bar is $20 \mu \mathrm{m}$.

coated core NPLs resulting in the increase of lateral dimensions from $38 \mathrm{~nm} \times 9 \mathrm{~nm}$ to $41 \mathrm{~nm} \times 10 \mathrm{~nm}$ for $1 \mathrm{ml}$ of CdS and $44 \mathrm{~nm} \times 11 \mathrm{~nm}$ for $2 \mathrm{ml}$ of CdS.

The reason for choosing the CdSe/2CdS NPLs as the fluorescence agents to fabricate the NPLs/PVP hybrid fibers is its higher QY when compared to the CdSe core and the CdSe/1CdS NPLs. Therefore, an approach was developed to prepare NPLs/PVP hybrid fibers with different NPLs contents, in which the trichloroethylene plays a vital role as a dispersing medium during the electrospinning process. SEM images of hybrid fibers with different contents of CdSe/2CdS NPLs were shown in Fig. 4. It can be seen that the ultrafine hybrid fibers were fabricated successfully; and the morphology of these hybrid fibers is similar to that of the original PVP fibers. These fibers are smooth and continuous with $2 \mu \mathrm{m}$ diameter. There was no significant change of the topography of these hybrid fibers, which indicates that the contents of NPLs have no obvious influence on the diameter of the hybrid fibers. The diameters of hybrid fibers became thicker slightly with the increase of NPLs content.

In order to demonstrate their fluorescence properties, the nanofiber networks obtained with different NPLs contents were characterized by microscopy under the UV light. Fig. 5 shows the microscopic images of CdSe/2CdS NPLs/PVP nanofiber networks on the glass substrates under the UV light. And the fiber networks exhibited strong and consequent red-color, which indicated that the NPLs were introduced homogeneously over the whole PVP fibers. Obviously, the NPLs content of $1 \mathrm{wt} \%$ was too small to get bright luminescence in doped sample (Fig. 5a), while the content of $3 \mathrm{wt} \%$ showed strong luminescence intensity (Fig. 5b). This indicates that as the NPLs contents increase, the luminescence intensity of the nanofiber network enhances.

Fig. 6 is a TEM image of the nanofibers. The existence and distribution of the NPLs can be clearly
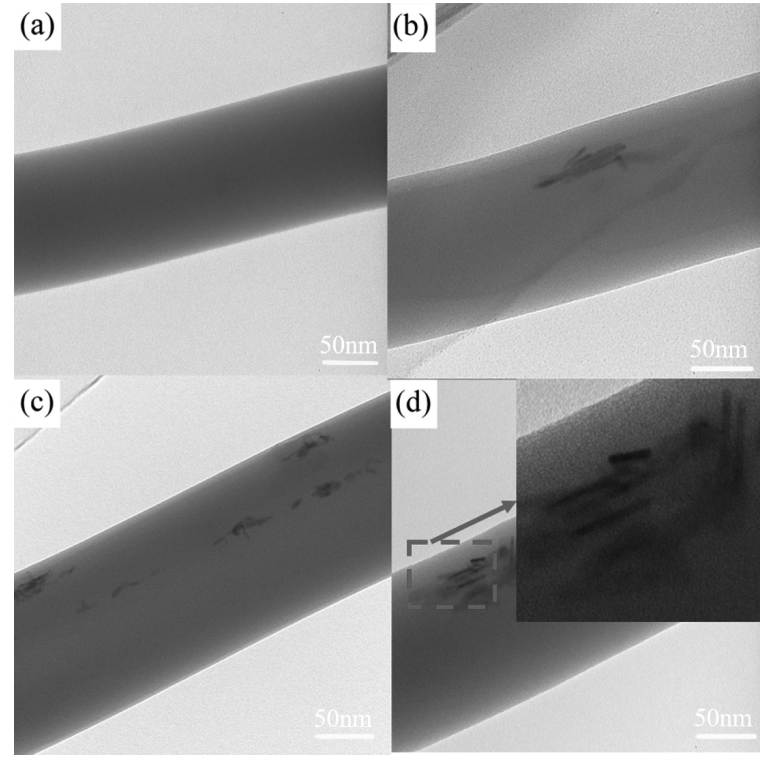

Fig. 6 TEM images of nanofibers with different NPLs contents: (a) $0 \mathrm{wt} \%$, (b) $1 \mathrm{wt} \%$, (c) $3 \mathrm{wt} \%$, (d) $5 \mathrm{wt} \%$.

seen in the figures. Obviously, zero doped nanofiber does not have a smooth surface (Fig. 6a), and all the doped nanofibers have the same smooth surfaces (Fig. 6b,c,d). The only difference between the two groups fibers is that there are NPLs inside the doped fibers. The spinning process did not destroy the morphology of the NPLs and maintained their excellent properties. The optical properties of the prepared NPLs/PVP nanofibers with NPLs content of $3 \mathrm{wt} \%$ have been further investigated and the nanofibers still remain the ideal fluorescence properties of the NPLs. As shown in Fig. 7, the absorption and emission of the CdSe/2CdS NPLs and nanofibers exhibited similar characteristics. The nanofibers exhibited the same first two excitonic transitions at the $572 \mathrm{~nm}$ and $618 \mathrm{~nm}$ (Fig. 7a), and a narrow emission peak at $628 \mathrm{~nm}$ (Fig. 7b), which had a slight red shift compared with the NPLs at $570 \mathrm{~nm}$ and $616 \mathrm{~nm}$ for absorptions (Fig. 7a) and at $624 \mathrm{~nm}$ for emission (Fig. 7b). It indicated that the emission peak was slightly redshift $(4 \mathrm{~nm})$ from the solution photoluminescence measured on the NPLs. The possible reason should relate to the slight additional energy relaxation due to coordination effect of oxygen atoms at carboxyl groups on PVP skeleton onto the surface of NPLs [31].

The functional groups of the PVP and nanofiber network were identified by FTIR spectra (Fig. 8). It could be seen that there was no obvious difference between the PVP and nanofiber network be- 

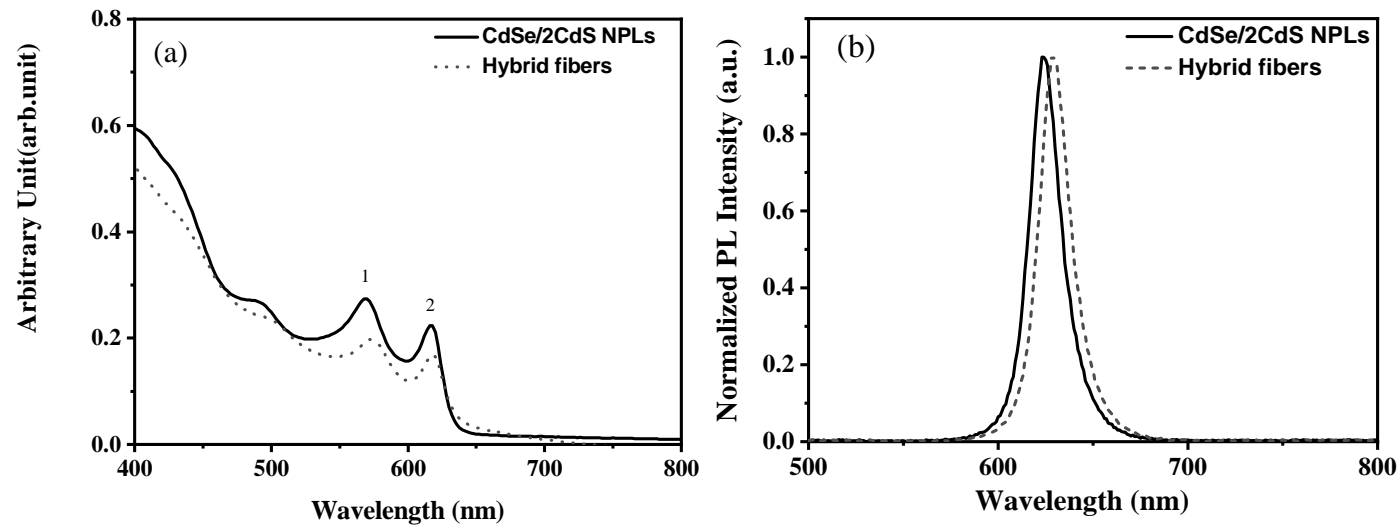

Fig. 7 (a) Absorption and (b) photoluminescence spectra of CdSe/2CdS NPLs and NPLs/PVP nanofibers. The PL emission spectra were collected with an excitation of $460 \mathrm{~nm}$.

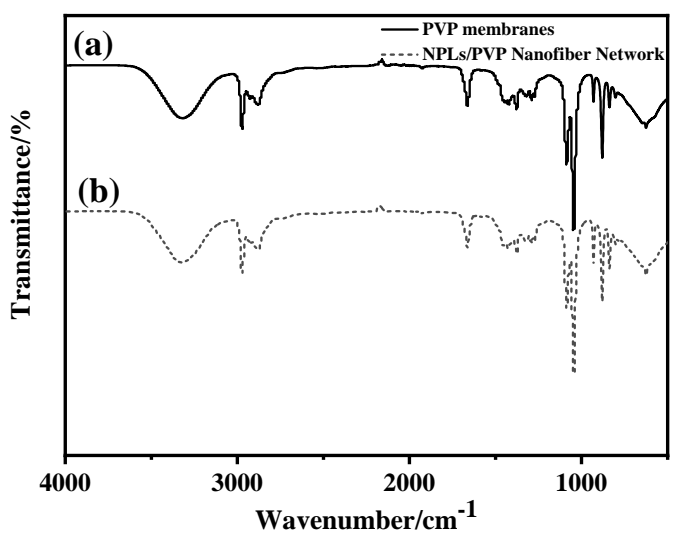

Fig. 8 FTIR spectra of the PVP and NPLs/PVP membranes: (a) PVP membranes, (b) NPLs/PVP nanofiber network.

cause the embedding content of NPLs was very low. There were a number of absorption characteristics of PVP observed in the FTIIR curves, the bond at $1663 \mathrm{~cm}^{-1}$ is assigned to the $\mathrm{C}=\mathrm{O}$ vibration of the amide groups, and the bond located at $1439 \mathrm{~cm}^{-1}$ and $1391 \mathrm{~cm}^{-1}$ were assigned with the cyclic $\mathrm{C}-\mathrm{H}$ structures of pyrrolidine in PVP [32]. Moreover, the peaks at $2982 \mathrm{~cm}^{-1}$ and $2875 \mathrm{~cm}^{-1}$ were related to the stretching vibration of $\mathrm{C}-\mathrm{H}$ and the broad band from $3583 \mathrm{~cm}^{-1}$ to $3081 \mathrm{~cm}^{-1}$ related to the overlap of the stretching vibrations of $\mathrm{O}-\mathrm{H}$ group with $\mathrm{N}-\mathrm{H}$ group [33].

\section{CONCLUSION}

Nanofiber networks, with a narrow photoluminescence based on the CdSe/CdS NPLs and PVP, have been fabricated via electrospinning technology for the first time, using oil-soluble NPLs as the fluorescence material and PVP as the matrix. The CdSe core and CdSe/CdS core-shell structure ware synthesized first, and then nanofiber networks of NPLs/PVP with different NPLs contents were fabricated by electrospinning. The as-prepared nanofiber network still maintained the excellent optical properties of the NPLs. As the content of NPLs increased, the photoluminescence intensity of nanofiber network enhanced. NPLs aggregation was occurred at the $5 \mathrm{wt} \%$ content.

Acknowledgements: This work was supported by (1) State Key Project of International Cooperation Research (2016YFE0110800, 2017YFE0108300);

(2) National Natural Science Foundation of China (51473082); (3) National Program for Introducing Talents of Discipline to Universities ("111" plan); (4) High-End Foreign Talent Project; (5) Double Hundred Foreign Expert Project of Shandong, China; (6) 1st Class Discipline Program of Materials Science of Shandong Province, China.

\section{REFERENCES}

1. Wood V, Panzer M, Caruge JM, Halpert J, Bawendi $\mathrm{M}$, and Bulovic V (2009) Air-stable operation of transparent, colloidal quantum dot based LEDs with a unipolar device architecture. Nano Lett 10, 24-29.

2. Zhang F, Wang S, Wang L, Lin Q, Shen $H$, Cao W, Yang C, Wang H, et al (2016) Super color purity green quantum dot light-emitting diodes fabricated by using CdSe/CdS nanoplatelets. Nanoscale 8, 12182-12188.

3. Lee $\mathrm{KH}$, Han CY, Kang HD, Ko H, Lee C, Lee J, Myoung N, Yim SY, et al (2015) Highly efficient, color-reproducible full-color electroluminescent devices based on red/green/blue quantum dot-mixed multilayer. ACS Nano 9, 10941-10949. 
4. Fan F, Kanjanaboos P, Saravanapavanantham M, Beauregard E, Ingram G, Yassitepe E, Adachi MM, Voznyy O, et al (2015) Colloidal CdSe(1-x)S(x) nanoplatelets with narrow and continuously-tunable electroluminescence. Nano Lett 15, 4611-4615.

5. Shirasaki Y, Supran GJ, Bawendi MG, Bulović V (2013) Emergence of colloidal quantum-dot lightemitting technologies. Nat Photonics 7, 13-23.

6. She C, Fedin I, Dolzhnikov DS, Dahlberg PD, Engel GS, Schaller RD, Talapin DV (2015) Red, yellow, green, and blue amplified spontaneous emission and lasing using colloidal CdSe nanoplatelets. ACS Nano 9, 9475-9485.

7. Chen Z, Nadal B, Mahler B, Aubin H, Dubertret B (2014) Quasi-2D colloidal semiconductor nanoplatelets for narrow electroluminescence. $A d v$ Funct Mater 24, 295-302.

8. Tessier M, Javaux C, Maksimovic I, Loriette V, Dubertret B (2012) Spectroscopy of single CdSe nanoplatelets. ACS Nano 6, 6751-6758.

9. Ithurria S, Tessier MD, Mahler B, Lobo RPSM, Dubertret B, Efros AL (2011) Colloidal nanoplatelets with two-dimensional electronic structure. Nat Mater 10, 936-941.

10. Lhuillier E, Pedetti S, Ithurria S, Nadal B, Heuclin H, Dubertret B (2015) Two-dimensional colloidal metal chalcogenides semiconductors: Synthesis, spectroscopy, and applications. Acc Chem Res 48, 22-30.

11. Tessier MD, Mahler B, Nadal B, Heuclin H, Pedetti S, Dubertret B (2013) Spectroscopy of colloidal semiconductor core/shell nanoplatelets with high quantum yield. Nano Lett 13, 3321-3328.

12. Empedocles SA, Norris DJ, Bawendi MG (1996) Photoluminescence spectroscopy of single CdSe nanocrystallite quantum dots. Phys Rev Lett 77, 3873-3876.

13. Roy D, Routh T, Asaithambi AV, Mandal S, Mandal PK (2016) Spectral and temporal optical behavior of blue-, green-, orange-, and red-emitting CdSebased core/gradient alloy shell/shell quantum dots: Ensemble and single-particle investigation results. $J$ Phys Chem C 6, 3483-3491.

14. Achtstein AW, Prudnikau AV, Ermolenko MV, Gurinovich LI, Gaponenko SV, Woggon U, Baranov AV, Leonov MY, et al (2014) Electroabsorption by OD, 1D, and 2D nanocrystals: A comparative study of CdSe colloidal quantum dots, nanorods, and nanoplatelets. ACS Nano 8, 7678-7686.

15. Kim J, Lee TS (2017) Emission tuning with sizecontrollable polymer dots from a single conjugated polymer. Small 14, ID 1702758.

16. Achtstein AW, Antanovich A, Prudnikau A, Scott R, Woggon U, Artemyev M (2015) Linear absorption in CdSe nanoplates: Thickness and lateral size dependency of the intrinsic absorption. J Phys Chem C 34, 20156-20161.
17. Wang Y, Huang L, Tang J, Wang Y, Wang W, Belfiore LA, Kipper MJ (2017) Melt spinning fibers of isotactic polyproplene doped with long-lifetime luminescent inorganic-organic $\mathrm{SiO}_{2}-\mathrm{Eu}^{3+}$ hybrid nanoparticles. Mater Lett 204, 31-34.

18. Rossinelli AA, Riedinger A, Marquésgallego P, Knüsel PN, Antolinez FV, Norris DJ (2017) High-temperature growth of thick-shell CdSe/CdS core/shell nanoplatelets. Chem Comm 53, 9938-9941.

19. Olutas M, Guzelturk B, Kelestemur Y, Yeltik A, Delikanli S, Demir HV (2015) Lateral size-dependent spontaneous and stimulated emission properties in colloidal CdSe nanoplatelets. ACS Nano 9, 5041-5050.

20. Chen BY, Kuo CC, Huang YS, Lu ST, Liang FC, Jiang DH (2015) Novel highly selective and reversible chemosensors based on dual-ratiometric fluorescent electrospun nanofibers with $\mathrm{pH}$ - and $\mathrm{Fe}^{3+}$-modulated multicolor fluorescence emission. ACS Appl Mater Interfaces 7, 2797-2808.

21. Putthithanad P, Rattanaumpa T, Pandhumas T, Budsombat S (2019) Sensitive 2,4-dinitrotoluene fluorescence sensors based on porous electrospun fibres and porous membranes. ScienceAsia 45, 36-42.

22. Zhou T, Zhu B, Chen F, Liu Y, Ren N, Tang J, Ma X, $\mathrm{Su} Y$, et al (2017) Micro-/nanofibers prepared via coassembly of paclitaxel and dextran. Carbohydr Polym 157, 613-619.

23. Qiao H, Xia Z, Fei Y, Cai L, Cui R, Cai Y, Wei Q, Yao Q (2017) Electrospinning combined with hydrothermal synthesis and lithium storage properties of $\mathrm{ZnFe}_{2} \mathrm{O}_{4}$-graphene composite nanofibers. Ceram Int 43, 2136-2142.

24. Guan Y, Li W, Zhang Y, Shi Z, Tan J, Wang F, Wang Y (2017) Aramid nanofibers and poly (vinyl alcohol) nanocomposites for ideal combination of strength and toughness via hydrogen bonding interactions. Compos Sci Technol 144, 193-201.

25. Li M, Zhang J, Zhang H, Liu Y, Wang C, Xu X, Tang Y, Yang B (2010) Electrospinning: A facile method to disperse fluorescent quantum dots in nanofibers without Förster Resonance Energy Transfer. Adv Funct Mater 17, 3650-3656.

26. Dai X, Zhang Z, Jin Y, Niu Y, Cao H, Liang X, Chen L, Wang J, et al (2014) Solution-processed, highperformance light-emitting diodes based on quantum dots. Nature 515, 96-99.

27. Demir MM, Soyal D, Ünlü C, Kuş M, Özçelik S (2009) Controlling spontaneous emission of CdSe nanoparticles dispersed in electrospun fibers of polycarbonate urethane. J Phys Chem C 113, 11273-11278.

28. Lu X, Zhao Y, Wang C (2010) Fabrication of PbS nanoparticles in polymer-fiber matrices by electrospinning. Adv Mater 17, 2485-2488.

29. Abitbol T, Wilson JT, Gray DG (2010) Electrospinning of fluorescent fibers from CdSe/ZnS quantum dots in 
cellulose triacetate. J Appl Polym Sci 119, 803-810.

30. Ithurria S, Talapin DV (2012) Colloidal atomic layer deposition (c-ALD) using self-limiting reactions at nanocrystal surface coupled to phase transfer between polar and nonpolar media. $\mathrm{J} \mathrm{Am} \mathrm{Chem} \mathrm{Soc}$ 134, 18585-18590.

31. Kuramochi Y, Nakagawa T, Yokoo T, Yuasa J, Kawai T, Hasegawa Y (2012) Eu(III) emission band changes caused by peripheral C-H/O hydrogen bonding. Dalton Trans 41, 6634-6640.

32. Zhou Z, Huang G, Xiong Y, Zhou M, Zhang S, Tang CY,
Meng F (2017) Unveiling the susceptibility of functional groups of polyethersulfone/polyvinylpyrrolidone membranes to NaOCl: A two-dimensional correlation spectroscopic study. Environ Sci Technol 51, 14342-14351.

33. Hanafi Y, Szymczyk A, Rabiller-Baudry M, Baddari K (2014) Degradation of poly (ether sulfone)/polyvinylpyrrolidone membranes by sodium hypochlorite: insight from advanced electrokinetic characterizations. Environ Sci Technol 48, 13419-13426. 